\title{
Effects of stimulus level on nonspectral frequency discrimination by human subjects *
}

\author{
Bryan E. Pfingst *, Lisa A. Holloway, Natee Poopat, Arohan R. Subramanya, \\ Melissa F. Warren, Teresa A. Zwolan
}

Kresge Hearing Research Institute, Department of Otolaryngology, University of Michigan Medical Center, Ann Arbor, Michigan 48109-0506, USA

Received 22 September 1993; Revision received 4 April 1994; Accepted 27 April 1994

\begin{abstract}
Frequency difference limens were determined as a function of reference-stimulus level for pulsatile electrical stimuli in 5 postlingually deaf human subjects with Nucleus- 22 cochlear implants and for sinusoidally amplitude-modulated acoustic white noise stimuli in 4 normal-hearing humans. Subjects were tested at levels throughout the dynamic range and extending to the lowest detectable levels. Response stability was measured over the course of 10 sessions. For electrical stimulation in the deaf ears, difference limens decreased as a function of level throughout much or all of the dynamic range of hearing. This result contrasts with the case for nonspectral acoustic stimulation of normal-hearing subjects, where nonspectral frequency difference limens were strongly affected by level only near the detection threshold. These data suggest differences in the acoustic and electrical response spaces that must be considered in the design of auditory prosthesis processors.
\end{abstract}

Key words: Auditory prosthesis; Cochlear implant; Electrical stimulation; Rate discrimination; Humans; Nonspectral frequency discrimination; Psychophysics

\section{Introduction}

In a deaf ear, the sensation of pitch can be induced by presentation of a periodic current waveform to electrodes located near the auditory nerve. Discrimination of changes in the frequency or repetition rate of the electrical signal is possible, though usually not with the same acuity with which normal-hearing human subjects can discriminate changes in the frequency of many acoustic signals. The difference in acuity for frequency changes is probably due, in part, to the absence, in the electrically stimulated deaf ear, of the normal, detailed place representation of the signal's spectrum. This hypothesis is supported by studies in normal-hearing subjects using acoustic signals in which the usable frequency information is represented only in

\footnotetext{
Some of the data in this paper were reported at the 1993 Midwinter Research Meeting of the Association for Research in Otolaryngology.

* Corresponding author. Fax: (313) 764-0014; Internet: BRYAN.PFINGST@MED.UMICH.EDU
}

the time domain (Formby, 1985). An acoustic signal commonly used for this purpose is sinusoidally amplitude-modulated broadband noise (SAM noise). Amplitude-modulated noise signals have also been used to study speech perception that is based only on the temporal information in the speech waveform and to compare the hearing of normal subjects to that of deaf subjects with cochlear implants (Van Tasell et al., 1987; 1992). The assumption underlying these studies is that the information content of the acoustic amplitudemodulated noise signals simulates the information content of the signal received by users of single-channel cochlear implants.

There are a number of obvious differences between a deaf ear stimulated electrically and a normal ear stimulated with amplitude-modulated noise signals, including differences in the number of nerve fibers remaining in the deaf ear, the condition of those remaining fibers, and the spatial extent of the nerve array that is stimulated by the respective signals. The acoustic waveforms are also obviously different from the electrical, the SAM noise signal being much less deterministic. In an earlier study, using non-human primates, we 
examined the consequences of the similarities and differences between nonspectral acoustic stimulation of normal cars and electrical stimulation of deaf ears on discrimination of changes in stimulus frequency (Pfingst and Rai, 1990). Specifically, we compared discrimination of the modulation frequency of acoustic SAM noise signals in a normal-hearing ear with discrimination of the frequency of electrical sinusoids in a deaf, implanted contralateral ear in the same subjects. At high levels of stimulation, frequency difference limens for these two stimuli were similar. However, the effects of level on the frequency discrimination ability were markedly different for the two conditions. Frequency difference limens for electrical stimulation of the deaf ears increased as reference-stimulus level decreased throughout the dynamic range. In the lower third to lower half of the dynamic range, difference limens were very large and some subjects were unable to make the discrimination at all (Pfingst and Rai, 1990; plus unpublished observations). In contrast, for acoustic stimulation in the normal-hearing ear, modulationfrequency difference limens increased little with decreasing level except within 10 to $20 \mathrm{~dB}$ of the detection threshold.

These differences in nonspectral frequency discrimination ability between normal-hearing ears and electrically stimulated deaf ears suggest that nonspectral frequency discrimination is affected by the condition of the stimulated ear and/or the waveform of the signal used to carry the frequency information. These differences have implications for design of processors for auditory prostheses, which seek an effective translation between the response space for the acoustic environment and the response space for electrically stimulated deaf ears. Thus, a better understanding of the differences between the electrical and acoustic response spaces will aid in the design and calibration of these processors.

The experiment reported here is an extension of the Pfingst and Rai (1990) experiment, using human subjects rather than non-human primates ${ }^{1}$. Previous studies with SAM noise in normal-hearing human subjects have shown little effect of level through most of the dynamic range (Pfingst, 1993). A study using electrical stimulation in deaf human subjects (Feinman and Wakefield, 1990) showed strong level effects for low-

\footnotetext{
${ }^{1}$ Procedures used with the human subjects were reviewed and approved by The Committee to Review Grants for Clinical Research and Investigation Involving Human Beings of the University of Michigan Medical School (Institutional Review Board) prior to the beginning of these experiments and were in accordance with the principles of the Belmont Report (1979) and the Declaration of Helsinki (1975).
}

frequency signals, similar to those observed in non-human primates. However, all of the studies cited above were conducted primarily in the middle three quarters of the dynamic range. In this experiment, we extend the levels of the acoustic and electrical signals to the lowest levels where the subjects could reliably detect them. It is in this region that the strongest effects of level on discrimination of SAM noise signals are seen. For the studies with electrical stimulation, we also tested subjects at the maximum comfortable level of stimulation. These experiments, like the Pfingst and Rai (1990) experiments, were conducted using a reference-stimulus frequency of $100 \mathrm{~Hz}$ and test stimuli at frequencics above $100 \mathrm{~Hz}$. This frequency region, near the voice fundamental, is the region for which rate coding is most commonly used in cochlear prosthetics.

\section{Methods}

\subsection{Subjects}

Data were collected from 5 postlingually deaf human subjects implanted with the Cochlear Corporation Nucleus-22 Cochlear Implant System (Clark, 1987; Skinner et al., 1991) and 4 human subjects with normal hearing. Details about the subjects are given in Table 1 .

\subsection{Equipment and stimuli for deaf-implanted subjects}

For the deaf-implanted subjects, stimuli were delivered by an implanted receiver-stimulator driven by a Mini Speech Processor (MSP) that was controlled by a Cochlear Corporation Dual Processor Interface (DPI). The output of the DPI was controlled by an IBM PC,

Table 1

Subject characteristics

\begin{tabular}{lcllll}
\hline Subject & Sex & Age & $\begin{array}{l}\text { Duration of } \\
\text { profound } \\
\text { deafness prior } \\
\text { to implantation } \\
\text { (years) }\end{array}$ & $\begin{array}{l}\text { Duration of } \\
\text { prosthesis use } \\
\text { prior to these }\end{array}$ & $\begin{array}{l}\text { Electrodes } \\
\text { experiments } \\
\text { (months) }\end{array}$ \\
\end{tabular}

Deaf-implanted subjects

$\begin{array}{llcccc}\text { B.C. } & \text { M } & 48 & 2 & 36 & 19-21 \\ \text { S.C. } & \text { F } & 54 & 0.5 & 24 & 19-21 \\ \text { M.K. } & \text { F } & 70 & 14.5 & 36 & 15-17 \\ \text { M.O. } & \text { M } & 67 & 46 & 60 & 13-15 \\ \text { B.T. } & \text { M } & 59 & 8 & 32 & 19-22\end{array}$

Normal-hearing subjects

B.B. F 22

L.B. $\quad$ M 22

B.P. $\quad$ M 49

A.S. $\mathbf{M} 21$ 
fitted with a Cochlear Corporation IF4 board, using Cochlear Corporation software and software developed locally.

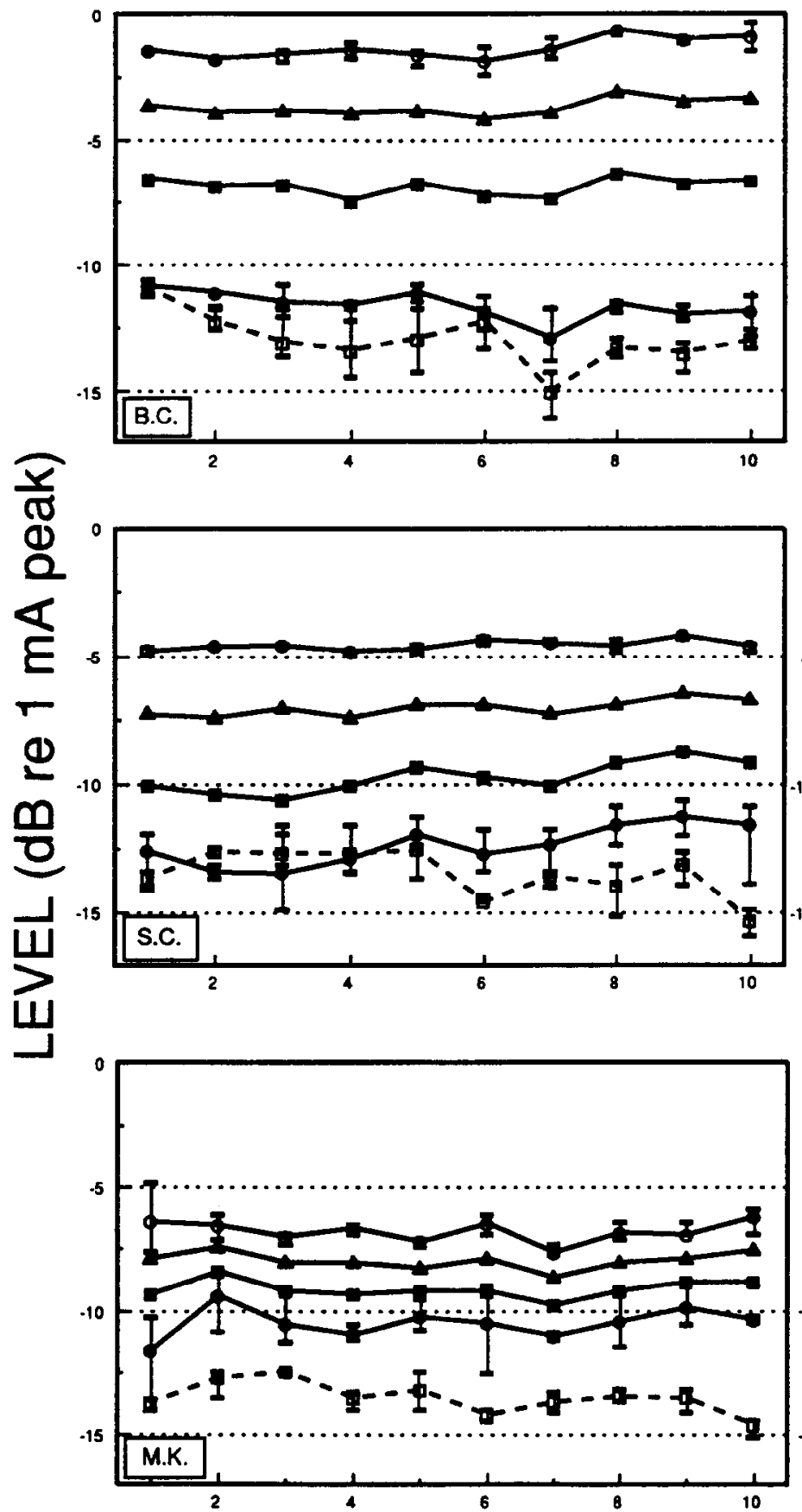

Temporal parameters of the stimuli were checked by monitoring the output of a VIPS receiver-stimulator, which was an external hardware device similar to
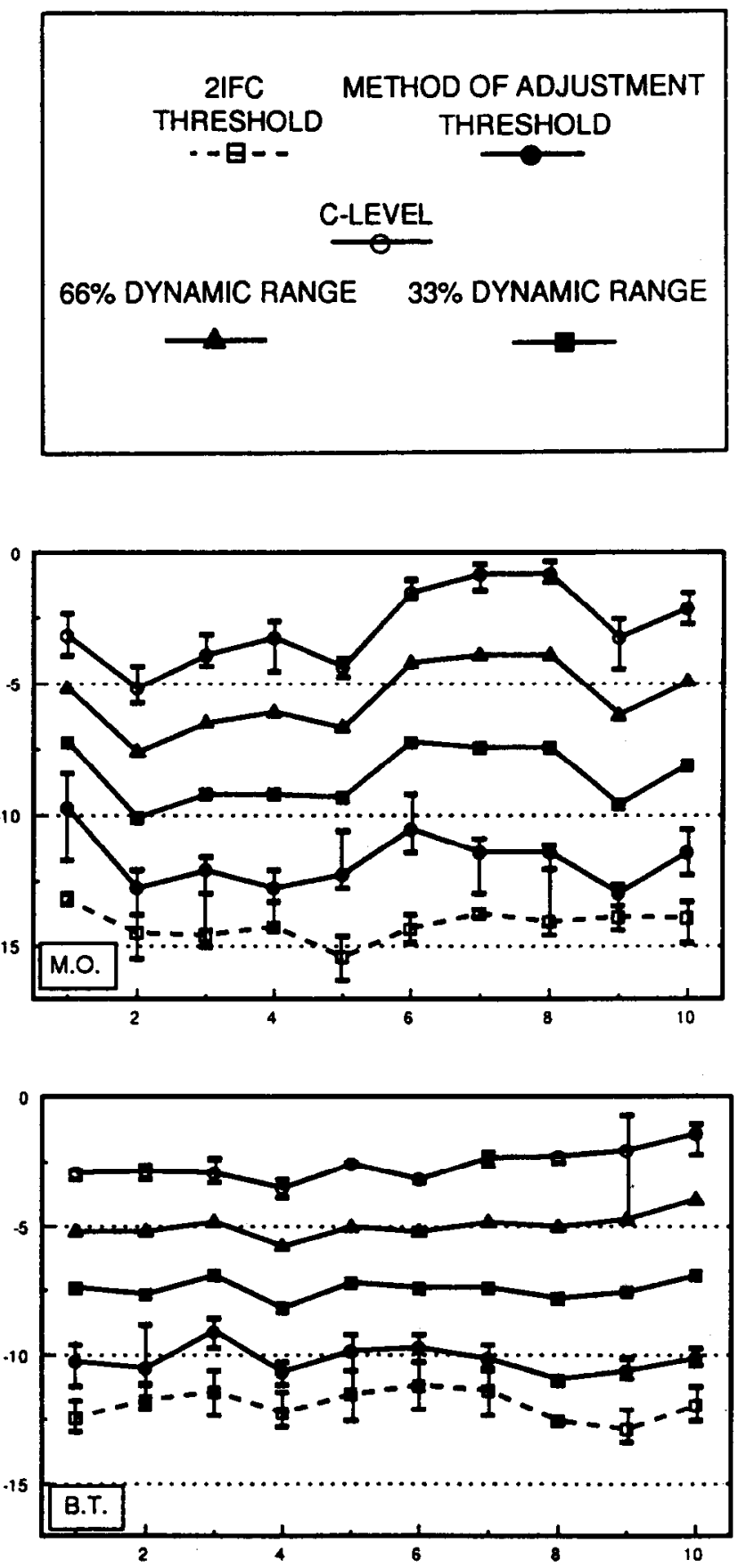

\section{SESSION NUMBER}

Fig. 1. Detection thresholds, maximum comfortable loudness levels (C-levels), and reference-stimulus levels for each of the 5 deaf-implanted subjects. Each panel shows data for a different subject. The legend (upper right) applies to all 5 subjects. Means and ranges for 3 estimates of threshold and C-level are shown for each of 10 sessions for each subject. Detection-threshold levels mcasurcd by the method of adjustment and the 2IFC method are shown. Derived reference-stimulus levels at 33 and $66 \%$ of the dynamic range are also shown for each session. These values, for each session, are based on the percent of the range, in current-level units, between the method-of-adjustment detection thresholds and the C-levels obtained at the beginning of that session. The percent-of-dynamic-range calculations were based on current-level units. The actual currents at these levels were then determined in $\mathrm{dB}$ re $1 \mathrm{~mA}$ peak, based on the current conversion charts for the subjects' implants. Frequency difference limens were measured at each of the 4 levels ranging from the method-of-adjustment detection threshold to the C-level (indicated by solid lines). 
the implanted receiver-stimulator. Voltage across a 30 $\mathrm{k} \Omega$ load at the output of the receiver-stimulator was monitorcd using a Tcktronix TDS 320 digital storage oscilloscope.

Any pulse started within the $200 \mathrm{~ms}$ specified stimulus duration was completed in order to achieve a charge-balanced signal. The stimulus duration (from the onset of the first pulse to the end of the last pulse) varied as a function of frequency, the maximum variation being equal to the stimulus period, which was a maximum of $10 \mathrm{~ms}$.

Current level for the cochlear implants was designated by numbers from 1 to 238 , which we termed 'current-level units.' The actual current in microamperes at each current-level unit was determined by Cochlear Corporation prior to implantation of each receiver-stimulator. Calibration tables from Cochlear Corporation were used to translate the current-level units to microamperes. The step size in decibels of current was variable from unit to unit, but the average step size was about $0.16 \mathrm{~dB}$ and at least $95 \%$ of step sizes were less than $0.45 \mathrm{~dB}$.

\subsection{Equipment and stimuli for normal-hearing subjects}

For normal-hearing subjects, acoustic stimuli were presented monaurally through a Bcycr Dynamic DT48 headphone fitted with a circumaural cushion (Pfingst et al., 1975) while subjects sat in a double-walled sound-attenuating booth (Tracoustics model RE-240B). The white noise carrier and the sinusoidal modulator for the SAM noise stimuli were generated by a General Radio white noise generator $(20 \mathrm{~Hz}-20 \mathrm{kHz})$ and a computer-controlled Rockland frequency synthesizer, respectively. These two signals were combined in a custom-built mixer to form the $100 \%$ amplitude-modulated SAM noise signal. The signal was gated by a custom-built tone switch (rise-fall times $=10 \mathrm{~ms}$ ) and attenuated by a computer-controlled Wilsonics (model PATT) attenuator. Experimental paradigms and data collection were controlled by IBM PC-compatible computers using programs written locally.

\subsection{Protocol for deaf-implanted subjects}

For the frequency discrimination experiments in the implanted subjects, only one electrode pair was tested (Table 1). The electrode configuration used was that which was normally used in the subject's speech processor. This configuration was 'bipolar plus one' (1.5 mm electrode separation, center to center) in all cases except subject B.T., where 'bipolar plus two' $(2.25 \mathrm{~mm}$ separation) was used. Selection of the electrode pair was restricted to electrodes in the apical half of the array (electrodes 12 to 22 ). Within this range, we chose the electrode pair with the largest dynamic range, based on detection thresholds and C-levels (maximum comfortable loudness levels) measured for each pair.

Four of the fivc implanted subjects participated in pilot experiments in which difference limens were measured at 25,50 , and $75 \%$ of the dynamic range based on detection thresholds and C-levels (in current-level units) obtained during the first session. During these experiments, 18 to 20 difference limens were obtained at each reference-stimulus level. Subject S.C. did not participate in these pilot experiments but was given a few practice sessions before beginning the main experiment.

In the main experiment, subjects were tested in sessions that lasted approximately 2 hours, using the following protocol. First, detection threshold levels and C-levels (maximum comfortable loudness levels) were determined for the electrode configuration selected for that subject. The stimuli were pulse trains at 100 pulses per second (pps), with a pulse duration of $204 \mu \mathrm{s}$ / phase and a stimulus duration of $200 \mathrm{~ms}$. Detection thresholds were determined using two different methods: a method of adjustment and a two-interval forced-choice (2IFC) method. In the method of adjustment, the subject used a current-level control knob to adjust the level of a repeating stimulus, with an interstimulus interval of $500 \mathrm{~ms}$, to a barely audible level. The 2IFC method was used in conjunction with a modified Levitt (1971) tracking procedure (Edwards and Wakefield, 1988 ) with a step size of 1 current-level unit. The last 10 reversals were used to determine thresholds at $70.7 \%$ correct responses. C-levels were measured using a method of adjustment. Subjects were asked to set the current-level control knob to 'the loudest position at which you can listen comfortably.' Three detectionthreshold determinations were made by each method, and three C-levels were determined. Means of the three determinations were calculated for each session.

Next, frequency difference limens were determined for the same electrode configuration using the $100 \mathrm{pps}$, $200 \mathrm{~ms}$ pulse train as the reference stimulus. Difference limens were determined at four different levels of the reference stimulus. The levels used were the mean of the three detection-threshold levels for that session determined by the method of adjustment, the mean of the three C-level determinations for that session, and levels corresponding to 33 and $66 \%$ of the range between these two levels in current-level units (Fig. 1). Frequency difference limens were measured using a 2IFC method with flanking cucs. The subject was presented on each trial with a sequence of four observation intervals signaled by lights. One of the two middle intervals in the sequence (either the second or the third, chosen at random) contained the test stimulus, which was higher in frequency (pulses per second) than the reference stimulus. The reference stimulus was presented during each of the other three intervals. The 
subject was asked to indicate in which interval ( 2 or 3 ) the stimulus sounded different. The modified Levitt tracking procedure (Edwards and Wakefield, 1988) was used with a ratio-step size of 1.3. The initial test stimulus was 300 pps. A correct response sequence caused the frequency value to be divided by 1.3 , and a miss caused it to be multiplied by 1.3 . The last 10 reversals were used to calculate the difference limen at $70.7 \%$ correct. Difference limens for the four levels were determined in random order before testing at any level was repeated. In each session, a total of three frequency difference limens were determined at each level.

In order to determine how frequency difference limens related to the loudness of the stimulus, loudness estimates were made for the reference stimuli ( $100 \mathrm{pps}$, $200 \mathrm{~ms}$ stimulus duration) in separate sessions. Detection thresholds and C-levels were measured at the beginning of the sessions, using the method of adjustment. The subjects were then presented a stimulus at the method-of-adjustment detection-threshold level and were told that this level was the softest level on a scale of 1 to 10 . They were then stimulated at the C-level and told that this was at level 10 . They were then stimulated at 7 to 9 different levels, including the detection threshold and the C-level, and asked to rank the loudness of each stimulus on a scale from 1 to 10 . The stimuli were presented in random order until a total of 10 trials were run at each level. Two to four sessions were run for each subject.

\subsection{Protocol for normal-hearing subjects}

For the normal-hearing subjects, the psychophysical testing consisted of two stages. In stage one, psychometric functions for stimulus detection were determined using a 2IFC procedure. SAM noise signals with a modulation frequency of $100 \mathrm{~Hz}$ were presented via the method of constant stimuli. A run consisted of 10 trials per stimulus level, and 3 runs were combined to determine a psychometric function. Detection threshold was defined as the level (calculated by linear interpolation) at which correct responses (hits) were obtained on $70.7 \%$ of the trials. Initial detection-threshold estimates were used to determine the levels at which the subject was to run in stage two.

In stage two, modulation-frequency difference limens for SAM noise were measured for each subject at 8 stimulus levels relative to the detection threshold. In order to concentrate on the subjects' abilities to discriminate between frequencies at levels close to the detection threshold, modulation-frequency difference limens were taken at $5,10,15,20,25,30,50$, and $70 \mathrm{~dB}$ SL, based on the initial detection-threshold estimates. Frequency difference limens were measured using a
2IFC procedure with two $200 \mathrm{~ms}$ bursts of SAM noise presented in each observation interval. In one interval, chosen at random on each trial, the modulation frequencies were identical $(100 \mathrm{~Hz})$, while in the other, the modulation frequencies differed $(100 \mathrm{~Hz}$ and a higher modulation frequency). The subjects were instructed to indicate the interval where the stimuli differed. A two-down, one-up tracking procedure with 20 reversals was used. Randomly interspersed catch-trials (i.e., trials in which the modulation frequencies of the stimuli in both observation intervals were identical) comprised approximately $20 \%$ of the stimuli in a run. These were intended to make the position in the tracking algorithm less salient to the subject. The difference limen was defined as the change in modulation frequency for which a subject was responding correctly on $70.7 \%$ of the trials. This analysis was restricted to test frequencies where eight or more trials were run. Each subject completed 10 to 12 runs for each of the 8 stimulus levels, and the difference limens from the last 5 of these runs were averaged in the final data analysis.

Detection-threshold tests were continued throughout the experiment, interspersed with the frequency discrimination testing. After all data collection was completed, a mean detection threshold was determined for each subject, based on detection thresholds measured over the same time period used for the frequency discrimination tests. These detection thresholds were used to determine the actual sensation levels at which the frequency discrimination data had been collected.

\section{Results}

\subsection{Deaf-implanted subjects}

\section{Operating ranges and reference-stimulus levels}

The four reference-stimulus levels used for frequency discrimination testing, and their stability over time, are shown in Fig. 1 (symbols connected by solid lines). Also shown in Fig. 1 are detection thresholds measured at the beginning of each session using the 2IFC method (open squares connected by dashed lines). The four reference-stimulus levels are based on method-of-adjustment detection thresholds (filled circles) and C-levels (open circles) measured at the beginning of each session. These two values were used as the highest and lowest reference-stimulus levels. Levels corresponding to 33 and $66 \%$ of the range between these values, in current-level units, comprised the remaining two reference-stimulus levels. These levels are plotted in Fig. 1 in units of $\mathrm{dB}$ re $1 \mathrm{~mA}$ peak.

Detection thresholds and C-levels varied from session to session. Standard deviations of the means across the 10 sessions averaged $0.94 \mathrm{~dB}$ for the method-of-ad- 
justment thresholds, $0.84 \mathrm{~dB}$ for the $2 \mathrm{IFC}$ thresholds, and $0.61 \mathrm{~dB}$ for the C-level measurements. Within subjects, the correlation of detcction thresholds (method of adjustment) with C-level measurements across sessions was generally low, averaging 0.25 .

For each subject the means of detection thresholds measured during the first 5 sessions were compared with the means of thresholds measured during the last
5 sessions. Differences between these two means averaged $-0.02 \mathrm{~dB}$ for the method-of-adjustment thresholds, $0.53 \mathrm{~dB}$ for the $2 \mathrm{IFC}$ thresholds, and $0.71 \mathrm{~dB}$ for the C-levels.

Detection thresholds measured with the 2IFC procedure were typically lower than those obtained by the method of adjustment. Across the 10 sessions, the average difference between detection thresholds ob-
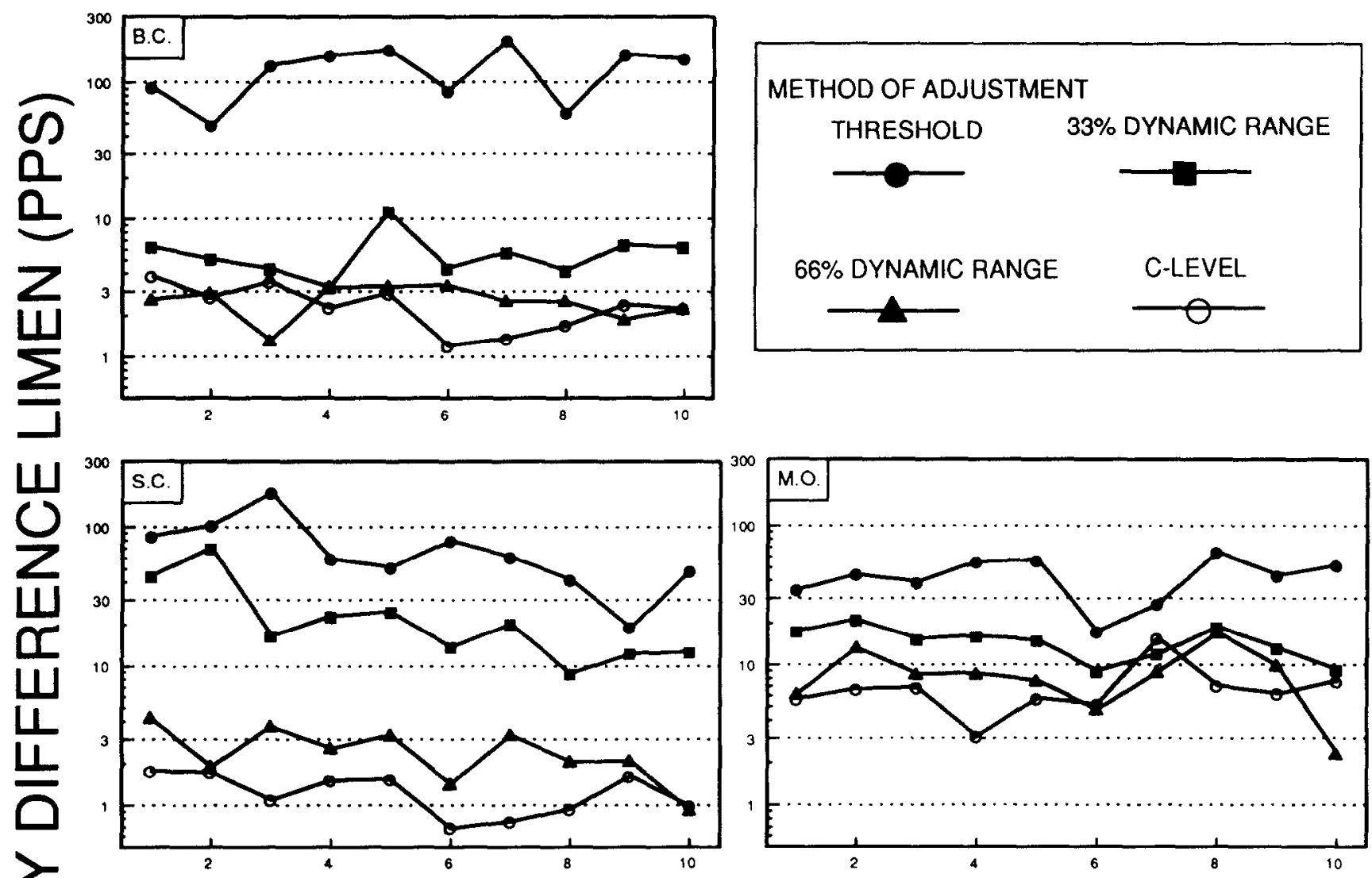

岂
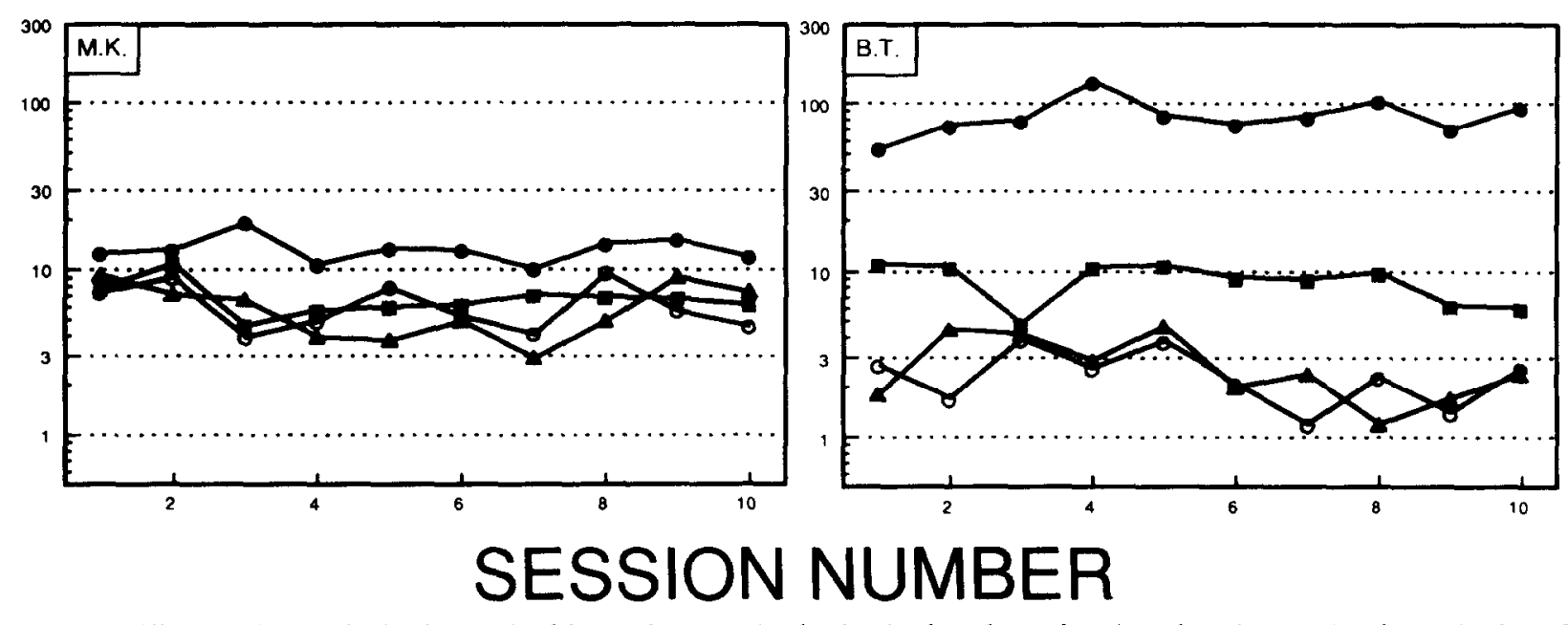

Fig. 2. Frequency difference limens obtained at each of four reference-stimulus levels plotted as a function of session number for each of the 5 deaf-implanted subjects. Each data point in the graphs represents the mean of 3 difference limen measurements made during the session. 
tained by these two methods ranged from $1.04 \mathrm{~dB}$ for subject S.C. to $3.02 \mathrm{~dB}$ for subject M.K. and averaged $1.91 \mathrm{~dB}$ across the 5 subjects.

\section{Stability over time}

Frequency difference limens for the 5 subjects are plotted as a function of session number in Fig. 2. Most of the subjects showed small decreases in the frequency difference limens over the course of the ten sessions. Subject S.C., who had the least amount of previous frequency discrimination training, showed the largest changes. In this subject, difference limens decreased by an average of $49 \%$ from the first 5 sessions to the last 5 sessions. The remaining 4 subjects showed changes from the first 5 to the last 5 sessions, averaged across the 4 levels, ranging from decreases of $24 \%$ (subject B.T.) to increases of $1.8 \%$ (subject M.O.). These subjects typically showed large difference limen decreases $(>20 \%)$ at only one or two levels. For example, subject B.T. showed decreases in mean difference limens from the first 5 sessions to the last 5 sessions of $>34 \%$ at the two highest levels but changes of $<1 \%$ at the lowest level.

Variation from session to session in frequency difference limens were not highly correlated with sessionto-session variation in reference-stimulus levels (average correlation coefficient $\mathbf{0 . 1 8 5}$ ), or C-levels (average correlation coefficient $=0.097$ ).

Difference limen vs level functions for the two subjects showing the greatest changes in difference limens over time are depicted in Fig. 3. For subject S.C., difference limens decreased from the first 5 sessions to the last 5 sessions at all reference-stimulus levels. The overall shape of the difference limen vs level function changed only slightly.

Subject B.T. showed decreases in the mean difference limens primarily at the highest two referencestimulus levels. These decreases resulted in a slightly steeper slope in the difference limen vs level function between the 33 and $66 \%$ of dynamic range referencestimulus levels, but the overall shape of the function did not change appreciably.

\section{Effects of reference-stimulus level}

Effects of reference-stimulus level on frequency difference limens are shown for all of the deaf-implanted subjects in Fig. 4. Since, as noted above, changes in difference limens over time had little effect on these functions, we averaged across all 10 sessions to obtain the values for Fig. 4. In Fig. 4A, the difference limens are plotted as a function of level in decibels of current. The location and extent of these functions on the abscissa indicate the magnitudes of detection thresholds and dynamic ranges in decibels of current, which varied from subject to subject. Subjects also differed in the magnitudes of their frequency difference limens

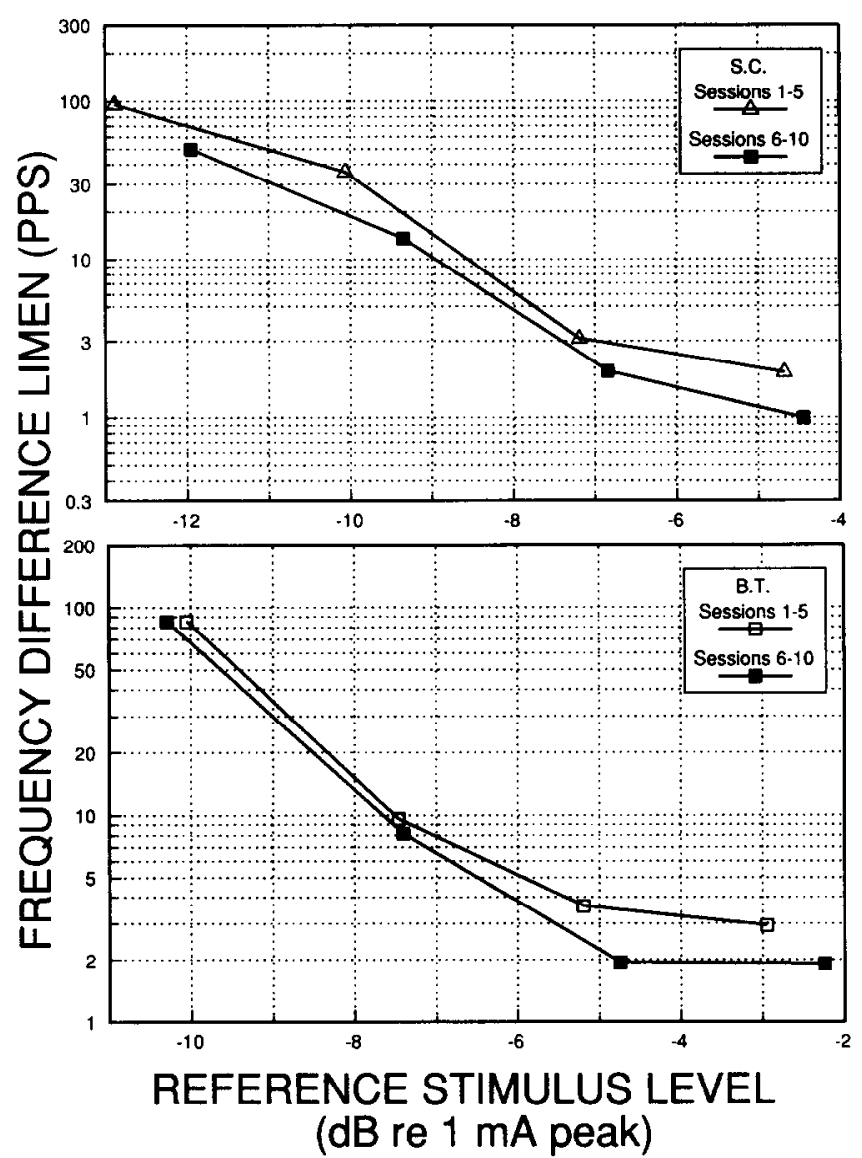

Fig. 3. Frequency difference limen vs level functions for subjects S.C. (upper graph) and B.T. (lower graph) based on blocks of 5 sessions each. Functions for the first 5 sessions (open symbols) are compared with those for the last 5 sessions (closed symbols).

and in the way in which frequency difference limens decreased as a function of level. The same data are plotted as a function of level in percent of the dynamic range in decibels of current in Fig. $4 \mathrm{~B}$ and as a function of sensation level in Fig. 4C. These depictions also illustrate the intersubject differences in the magnitudes of the difference limens and the rates of change in the difference limens as a function of reference-stimulus level.

For subjects S.C. and M.O., difference limens decreased as a function of level throughout the dynamic range, with a slight tendency toward asymptote. For subjects B.C. and B.T., difference limens decreased up to about $70 \%$ of the dynamic range and then showed a clear asymptote. Subject M.K. showed the shallowest functions, with difference limens decreasing little above $40 \%$ of the dynamic range.

\section{Loudness estimates}

Loudness estimates for stimuli ranging from the detection threshold to the C-level are shown in Fig. 5. This figure is based on data from one session in which method-of-adjustment detection thresholds and C- 
levels were determined at the beginning of the session and then loudness estimates were made based on the operating range bounded by those valucs. This procedure was repeated one to three times for each subject with comparable results. These functions show a slow growth of estimated loudness as a function of level

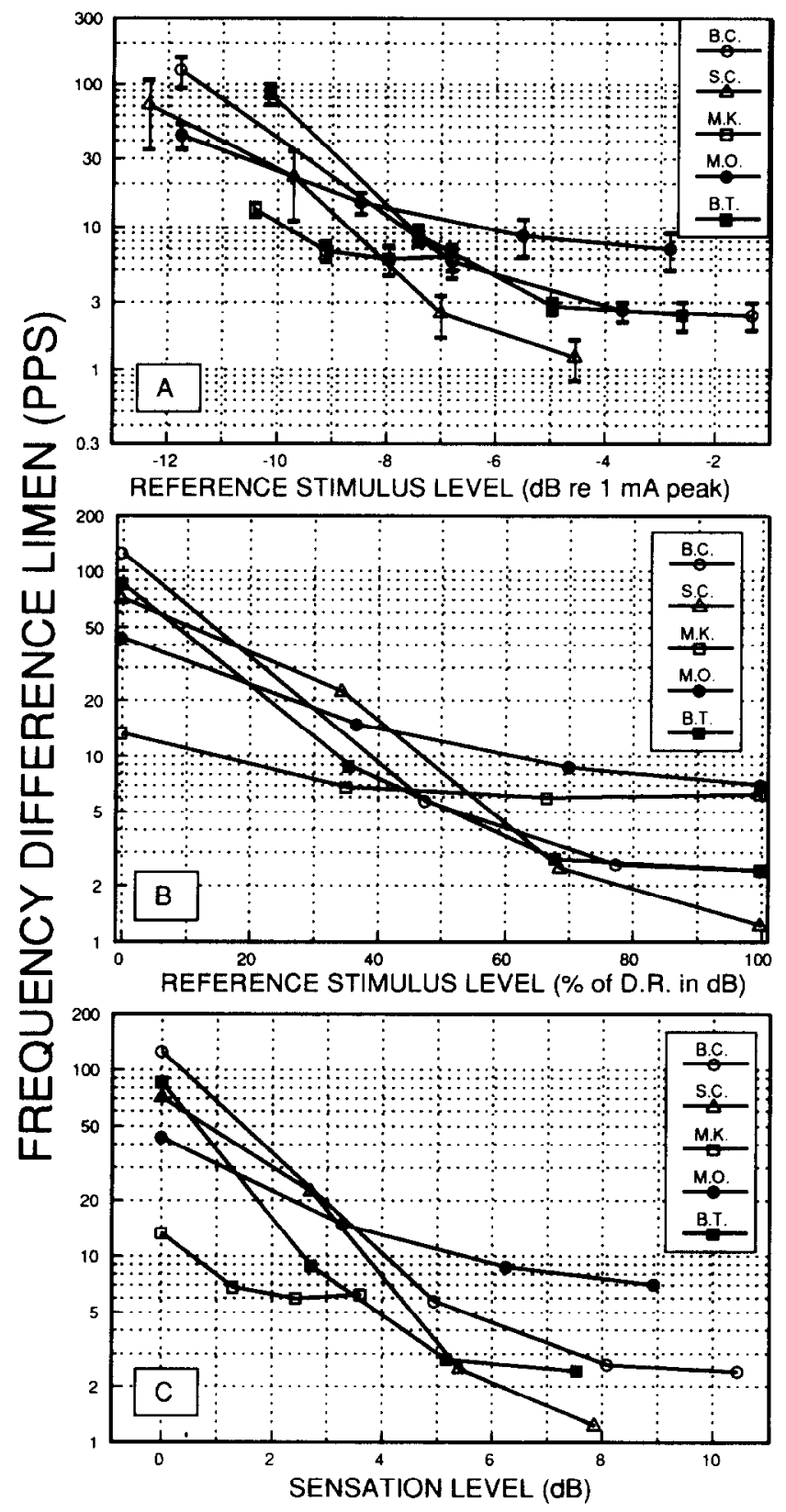

Fig. 4. Mean frequency difference limens for the 10 sessions for each deaf-implanted subject plotted as a function of the mean referencestimulus level for the 10 sessions. Three different representations of reference-stimulus level are used: (A) $\mathrm{dB}$ re $1 \mathrm{~mA}$ peak; (B) percent of dynamic range, where the dynamic range is in decibels of current; and $(C)$ sensation level. The thresholds and dynamic ranges used in (B) and (C) were based on method of adjustment. In (A), means and $95 \%$ confidence intervals are shown.

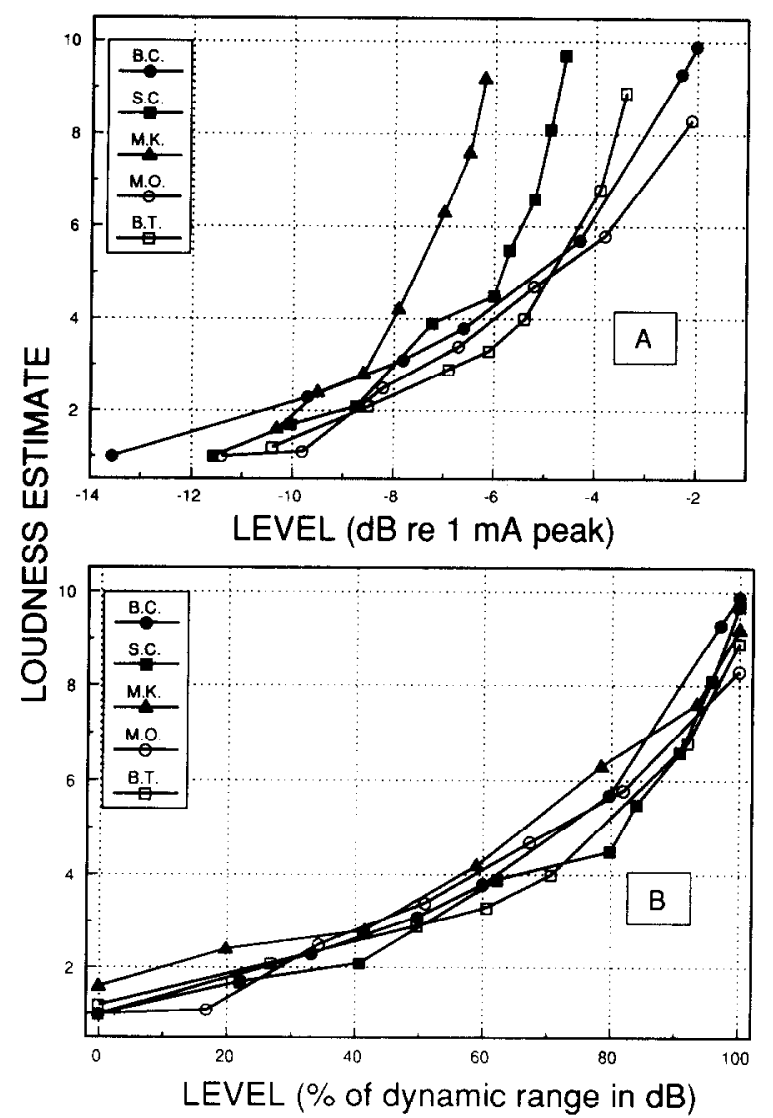

Fig. 5. Loudness estimate vs level functions for the 5 deaf-implanted subjects. Mean loudness estimates from 10 trials/level are plotted as a function of level. Two different representations of the stimulus level are used: (A) dB re $1 \mathrm{~mA}$ peak; and (B) percent of dynamic range, where the dynamic range is in decibels of current. The dynamic ranges used in (B) were based on method-of-adjustment detection thresholds and $\mathrm{C}$-levels measured at the beginning of the session.

near the detection threshold, with increasing rates of growth as level increases.

The frequency difference limen vs level data are replotted as difference limen vs estimated loudness functions in Fig. 6. This depiction indicates that the largest decreases in the frequency difference limens as a function of level occurred in the lower third of the range of estimated reference-stimulus loudness.

\subsection{Normal-hearing subjects}

\section{Stimulus detection}

Psychometric detection-threshold functions for runs conducted during stage two are shown in Fig. 7. Detection thresholds $(70.7 \%$ hits $)$ for the four subjects spanned a $4.4 \mathrm{~dB}$ range. The lowest level at which subjects detected the signal on $100 \%$ of the trials was an average of $8.0 \mathrm{~dB}$ (s.d. $=1.5 \mathrm{~dB}$ ) above the detection 


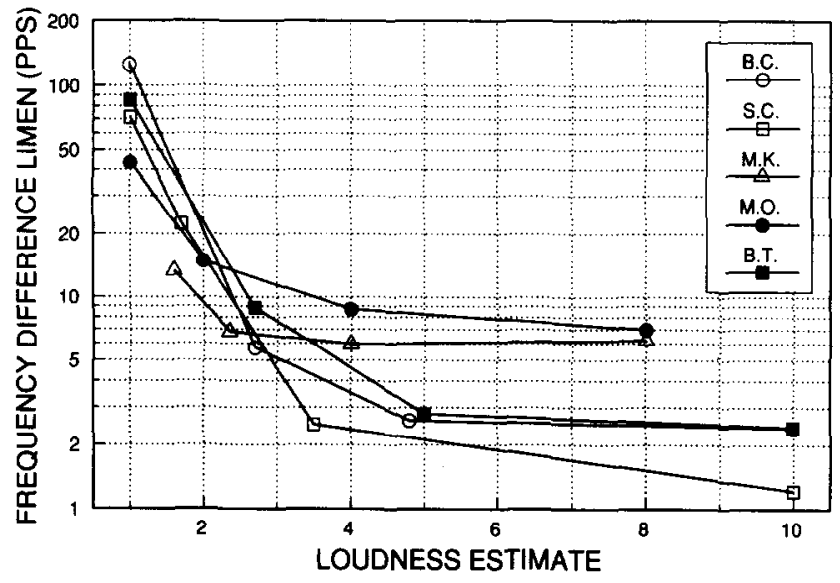

Fig. 6. Mean frequency difference limens for the 10 sessions for each of the 5 deaf-implanted subjects plotted as a function of the estimated loudness of the reference stimulus as determined from each subject's mean loudness estimate vs level function.

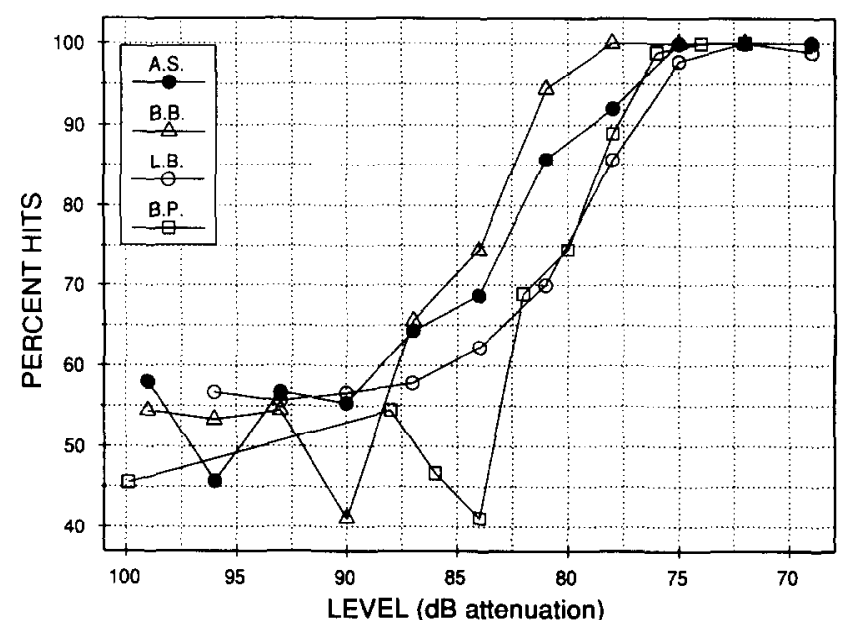

Fig. 7. Psychometric functions for stimulus detection for each of the 4 normal-hearing subjects. $100 \mathrm{~Hz}$ SAM noise stimuli were presented via a 2 IFC procedure using the method of constant stimuli. Each plot gives the average values of 9 sessions distributed evenly throughout stage two.
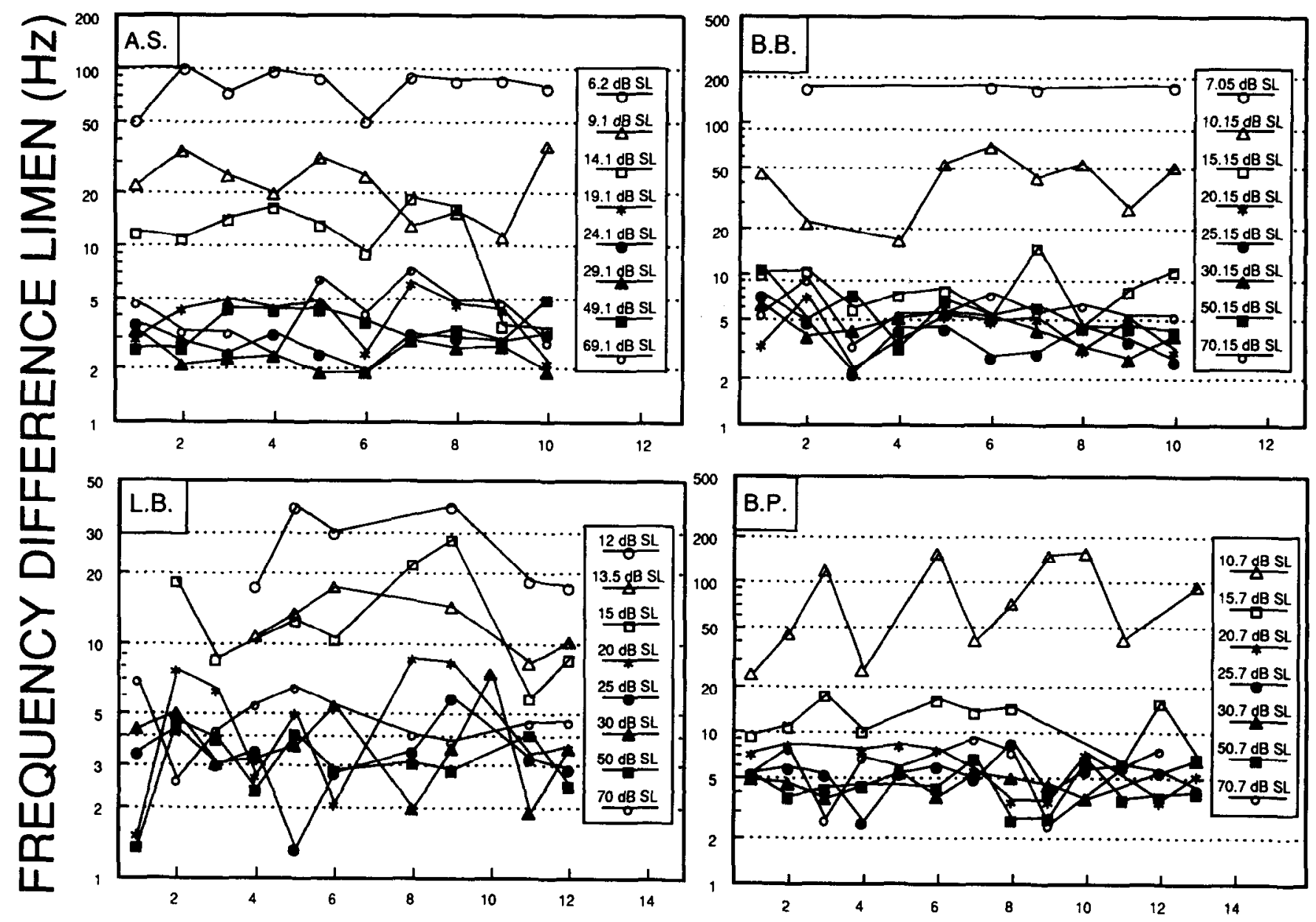

SESSION NUMBER

Fig. 8. Modulation-frequency difference limens plotted as a function of session number for each of the 4 normal-hearing subjects. Difference limens were determined using a 2 IFC procedure with a two-down, one-up tracking method. 


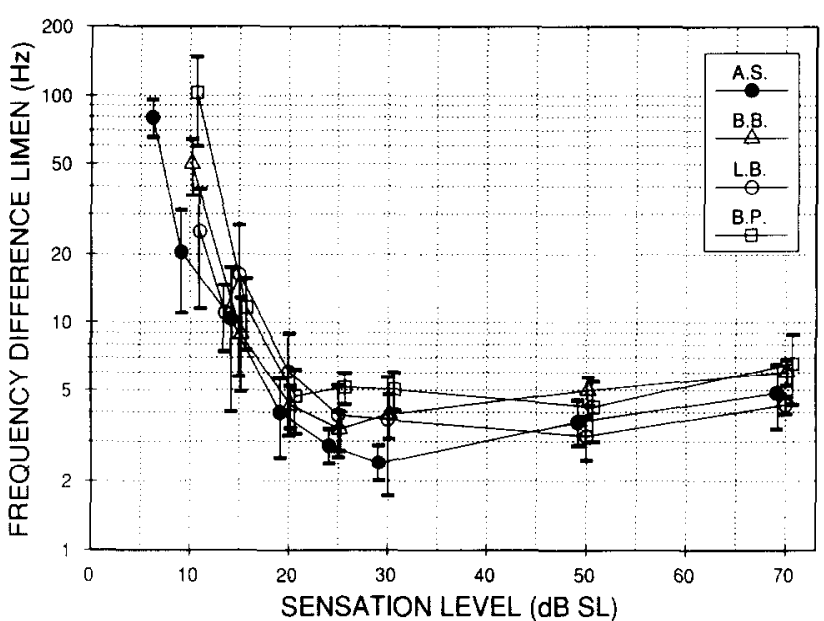

Fig. 9. Modulation-frequency difference limens for each of the 4 normal-hearing subjects plotted as a function of sensation level of the reference stimulus. Each data point gives the mean for 5 frequency difference limen measurements. Error bars indicate the $95 \%$ confidence intervals.

threshold and spanned a $6 \mathrm{~dB}$ range across the four subjects.

\section{Stability over time}

Frequency difference limens obtained at the eight stimulus levels are shown as a function of session number in Fig. 8. In general, we did not observe any systematic changes over time in the frequency difference limens of the four subjects. Standard deviations for the frequency difference limens obtained at sensation levels higher than $20 \mathrm{~dB}$ SL were less than 0.2 times their respective mean limens for three of the subjects and less than 0.36 times the mean for the fourth. Below $20 \mathrm{~dB}$ SL, the variability in the difference limens collected for each subject was larger: $0.3-$ 0.6 times the respective means. This was particularly true for difference limens measured at levels between 10 and $15 \mathrm{~dB}$ SL.

Subject L.B. had a cold at one point in the study, during session 7-10. The difference limens obtained in these runs were somewhat higher than those from the runs both preceding and following them. These sessions were included in Fig. 8, but they were not used to calculate the final mean difference limens.

\section{Effects of reference-stimulus level}

In Fig. 9, modulation-frequency difference limens for the four subjects are plotted as a function of sensation level of the reference stimulus. The difference limens of all of the subjects were largest when the level of SAM noise was closest to the detection threshold. As the level of the stimulus was increased to between 20 and $30 \mathrm{~dB}$ SL, the modulation-frequency difference limens for each subject decreased rapidly as a function of level. Subjects B.B. and A.S. showed 2 to $2.5 \mathrm{~Hz}$ increases in their modulation-frequency differ- ence limens as the SAM noise level was increased from $30 \mathrm{~dB}$ SL to $70 \mathrm{~dB}$ SL. Subjects L.B. and B.P. showed mostly continuous decreases in their difference limens up to about $50 \mathrm{~dB}$ SL. When the level of SAM noise was raised from $50 \mathrm{~dB}$ SL to $70 \mathrm{~dB}$ SL, these two subjects showed increases of 1 to $2 \mathrm{~Hz}$ in their difference limens.

Mean modulation-frequency difference limens could not be determined at $5 \mathrm{~dB}$ above the initial detection threshold for three out of the four subjects, because these subjects were not able to consistently discriminate between frequencies presented at this level. When we were able to obtain a difference limen for these subjects, their mean difference limens were at about $300 \mathrm{~Hz}$ (B.P.), $175 \mathrm{~Hz}$ (B.B.), and $90 \mathrm{~Hz}$ (L.B.). Instead of having these subjects run at levels where they were not able to consistently discriminate between frequencies, they each ran at the lowest levels at which we were able to reliably obtain difference limens. These stimulus levels were 10.7, 7.1, and $12 \mathrm{~dB}$ SL for subjects B.P., B.B., and L.B., respectively. These levels were all within $4 \mathrm{~dB}$ of the lowest level on the psychometric functions where the subject obtained $100 \%$ hits.

\section{Discussion}

The principal finding of this study was that nonspectral frequency difference limens for electrical stimulation of the deaf ear are affected by level over much or all of the dynamic range of hearing, while those for nonspectral acoustic stimulation of normal-hearing ears are affected primarily near the detection threshold. This finding confirms in human subjects the results previously obtained by Pfingst and Rai (1990) in monkeys, but it differs from that study in several important respects, as detailed below.

In this study, we extended the range of levels over which the subjects were tested. The human subjects in this study were able to perform the discrimination at lower levels in the dynamic range than were most of the monkey subjects tested in the Pfingst and Rai experiment. It remains to be determined whether this difference was due to species or some other difference, such as nerve survival pattern or the use of pulsatile versus sinusoidal stimuli. In addition, the range over which difference limens for electrical stimuli were tested in the current experiment was extended to the upper limits of the dynamic range. In this case, we saw some evidence in all subjects of an asymptote in the difference limen vs level functions.

Another difference between this study and the Pfingst and Rai experiment was in the waveform used for the electrical stimulation. In the former experiment, we used electrical sinusoids. Changing the frequency of those signals can produce marked changes in loudness. 
However, the effects of frequency on loudness are minimal for pulsatile electrical stimuli if the pulse durations arc less than about $0.5 \mathrm{~ms} /$ phasc (Shannon, 1985; Pfingst and Morris, 1993). In this study, we used $0.204 \mathrm{~ms}$ / phase pulsatile stimuli, which are the pulses commonly used in the subjects' wearable speech processors. Changes in the pulse rate over the range where we tested frequency difference limens would be expected to produce little, if any, change in loudness. While we cannot state that loudness cues never contributed to the pulse-rate discrimination, it seems highly unlikely that they dominated the discrimination, as they might well have done if sinusoids or long-duration pulses were used (Pfingst and Rush, 1987; Barretto and Pfingst, 1992).

A potential complication of using pulsatile stimuli is that the stimulus duration (from the onset of the first pulse to the end of the last pulse) changes as a function of pulse rate. In our experiments, the maximum difference in stimulus duration between the reference and test stimuli was less than $10 \mathrm{~ms}$ or less than $5 \%$ of the total stimulus duration. Stimulus duration difference limens for electrical stimuli are typically greater than $20 \%$ of the stimulus duration (Hochmair-Desoyer et al., 1985). Furthermore, in the current experiment, the stimulus duration differences associated with frequency differences that the subjects could not detect werc as large as those associated with frequency differences which they could detect. Thus, it is unlikely that duration changes were a useful cue in these experiments.

An important feature of the data obtained in the present study is that the across-subject differences in the shapes and slopes of the difference limen vs level functions for electrical stimulation were greater in the electrically-stimulated deaf-implanted subjects than in the acoustically-stimulated normal-hearing subjects. This feature was also seen in the Pfingst and Rai (1990) study. We saw even grcater across-subject variance in the deaf-implanted human subjects in our pilot studies. Those studies included a subject who had very steep difference limen vs level functions, but that subject was not able to participate in the main experiment.

Large effects of level on detection of periodicity (temporal modulation transfer functions) by deaf-implanted subjects have also been seen (Shannon, 1992, 1993). These results also contrast with the case for acoustic stimulation of the normal ear, where effects of level are seen only near the detection threshold (Vicmcister, 1979).

The large effects of level on electrical frequency difference limens, which are not seen when the normal auditory system is stimulated with nonspectral acoustic stimuli, might be due, in part, to the significant nerve loss that accompanies deafness (Hinojosa and Marion, 1983). Also, differences in nerve loss across subjects might account for the differences in performance across subjects. The assumption here is that nonspectral frequency difference limens are inversely related to the number of nerve fibers carrying the auditory information. As level increases and the number of fibers stimulated by the electrical signal grows, the difference limens decrease until some saturation level is reached, above which recruiting additional fibers has no further effect. In contrast, in the normal acoustic case, where the number of fibers stimulated at low levels is large, the difference limens for SAM noise stimuli reach a saturation level at only a few $\mathrm{dB}$ above the detection threshold.

Frequency discrimination tasks can be subject to large learning effects. These effects have been demonstrated primarily for pure tones (Turner and Nelson, 1982; Prosen et al., 1990). Large improvements in frequency difference limens for electrical stimulation of the ear were seen over the first two years following implantation in a study by Risberg and Agelfors (1987). These changes, however, were for a restricted group of subjects that were suspected to have some residual hair cells and electrophonic hearing (Risberg et al., 1990). The learning effects seen over the course of our experiments were small and had little effect on the main conclusions of the experiment. Thus, our results seem reasonably stable, though we cannot rule out significant cffects from very long-term training.

Small fluctuations in detection thresholds and Clevels were seen within subjects from session to session. Under our procedure for the frequency discrimination tests in the deaf-implanted subjects, these fluctuations resulted in small changes in the reference-stimulus levels from session to session. In general, these changes seemed to have little effect on difference limens, as evidenced by the low correlations across sessions between detection thresholds or C-levels and difference limens. We note that one of the subjects who showed moderately large decreases in difference limens over time (S.C.) also showed a slight increase in mean detection thresholds and C-levels from the first 5 to the last 5 sessions. The resulting changes in referencestimulus levels, however, were not sufficient to completely account for the decrease in difference limens observed over this time period. Comparable changes in reference-stimulus level during either the first 5 or the second 5 sessions did not result in as large a change in the frequency difference limen as was seen over time between the first 5 and last 5 sessions (Fig. 3).

Comparison of level effects for acoustic and electrical stimulation is complicated by the fact that the dynamic ranges for the two signals, and the growth of loudness within those ranges, are markedly different. If the frequency difference limen vs level functions for these two types of stimulation are compared in terms of sensation level, the functions show more similar behavior in that difference limens decrease rapidly as a 
function of level over the first $10-20 \mathrm{~dB}$ above threshold. However, $20 \mathrm{~dB}$ exceeded the entire dynamic rangc for our electrically stimulated subjects but is only about $25 \%$ of the dynamic range for acoustic stimulation of the normal ear. Loudness growth functions for the deaf-implanted subjects in our experiment, assessed by loudness estimation techniques, showed slow loudness growth as a function of decibels of current near the detection threshold and more rapid growth at higher levels, similar to functions reported previously for electrical stimulation (Pfingst, 1984; Shannon, 1985; Zeng and Shannon, 1992). Thus, the region where frequency difference limens are changing most rapidly as a function of reference-stimulus level in most subjects corresponds to the level where loudness is growing most slowly as a function of level. For these subjects, if frequency difference limens are plotted as a function of reference-stimulus loudness, it is evident that difference limens are affected by level primarily at the lowest loudnesses.

This study supports the previous finding of differences in the processing of frequency information by an electrically stimulated, deafened auditory system and processing of nonspectral acoustic information by a normal system. An appreciation of these differences may be useful in designing processors for auditory prostheses. In particular, it seems that there may be an advantage in compressing the range of levels onto which the lower part of the acoustic dynamic range is mapped and expanding the mapping of the upper part of the range. Such a mapping would also correct for the unusually slow growth of loudness that is commonly seen near the detection threshold in the case of electrical stimulation of the deaf ear.

More work is needed to determine the mechanisms underlying the differences between the electrically stimulated deaf ear and the acoustically stimulated normal ear. If these differences arc duc, in part, to differences in nerve survival patterns, then the frequency discrimination data may be useful in diagnosing the condition of the deaf ear and in determining the best strategy for mapping the acoustic space to the electrical space on a patient-by-patient basis.

\section{Acknowledgements}

This research was supported by Grant No. DC00560 from the National Institute on Deafness and Other Communication Disorders at the National Institutes of Health. We express appreciation to Gregory Wakefield and Paul Kileny for the use of their testing facilities and to Gail Feinman and Leslie Collins for software development.

\section{References}

Barretto, R.L. and Pfingst, B.E. (1992) Electrical stimulation of the auditory nerve: Effects of pulse width on frequency discrimination. Hear. Res. 62, 245-249.

Belmont Report: The National Commission for the Protection of Human Subjects of Biomedical and Behavioral Research. (1979) Ethical principles and guidelines for the protection of human subjects of research. Federal Register, Washington, DC.

Clark, G.M. (1987) The University of Melbourne - Nucleus multielectrode cochlear implant. Adv. Otorhinolaryngol. 38, 1-189.

Declaration of Helsinki (1975) Recommendations guiding medical doctors in biomedical research involving human subjects, adopted by the 18th World Medical Assembly, Helsinki, Finland, 1964 and revised by the 29th World Medical Assembly, Tokyo, Japan, 1975.

Edwards, B.W. and Wakefield, G.H. (1988) Small-sample statistical analysis of Levitt's psychophysical procedure. J. Acoust. Soc. Am. $83, \mathrm{~S} 17$.

Feinman, G.R. and Wakefield, G.H. (1990) The effects of amplitude on pulse rate discrimination under electrical stimulation. Abstr. 2nd Int. Coch. Implant Symp., June, 1990, Iowa City, p. 43.

Formby, C. (1985) Differential sensitivity to tonal frequency and to the rate of amplitude modulation of broadband noise by normally hearing listeners. J. Acoust. Soc. Am. 78, 70-77.

Hinojosa, R. and Marion, M. (1983) Histopathology of profound sensorineural deafness. Ann. NY Acad. Sci. 405, 459-484.

Hochmair-Desoyer, I.J., Hochmair, E.S. and Stiglbrunner, H.K. (1985) Psychoacoustic temporal processing and speech understanding in cochlear implant patients. In: R.A. Schindler and M.M. Merzenich (Eds.), Cochlear Implants, Raven Press, New York, pp. 291-304.

Levitt, H. (1971) Transformed up-down methods in psychoacoustics. J. Acoust. Soc. Am. 49, 467-477.

Pfingst, B.E. (1984) Operating ranges and intensity psychophysics for cochlear implants. Implications for speech processing strategies. Arch. Otolaryngol. Head. Neck. Surg. 110, 140-144.

Ptingst, B.E. (1993) Comparison of spectral and nonspectral frequency difference limens for human and nonhuman primates. J. Acoust. Soc. Am. 93, 2124-2129.

Pfingst, B.E., Hienz, R., Kimm, J. and Miller, J.M. (1975) Reactiontime procedure for measurement of hearing. I. Suprathreshold functions. J. Acoust. Soc. Am. 57, 421-430.

Pfingst, B.E. and Morris, D.J. (1993) Stimulus features affecting psychophysical detection thresholds for electrical stimulation of the cochlea. II: Frequency and interpulse interval. J. Acoust. Soc. Am. 94, 1287-1294.

Pfingst R.F. and Rai, D.T. (1990) Effects of level on nonspectral frequency difference limens for electrical and acoustic stimuli. Hear. Res. 50, 43-56.

Pfingst, B.E. and Rush, N.L. (1987) Discrimination of simultaneous frequency and level changes in electrical stimuli. Ann. Otol. Rhinol. Laryngol. (St. Louis, MO) 96, 34-37.

Pfingst, B.E., Warren, M.F., Poopat, N. and Zwolan, T.A. (1993) Electrical stimulation of the cochlea: Effects of stimulus level on frequency discrimination by human and nonhuman primates. Abstr. Assoc. Res. Otolaryngol. 77.

Prosen, C.A., Moody, D.B., Sommers, M.S. and Stebbins, W.C. (1990) Frequency discrimination in the monkey. J. Acoust. Suc. Am. 88, 2152-2158.

Risberg, A. and Agelfors, E. (1987) The relation between speech perception ability and results on psychoacoustic tests for single channel cochlear implants. In: P. Banfai (Ed.), Cochlear Implant: Current Situation, Bermann, Erkelenz, FRG, pp. 187-191.

Risberg, A., Agelfors, E., Lindström, B. and Bredberg, G. (1990) Electrophonic hearing and cochlear implants. Acta Otolaryngol. (Stockholm), 156-163. 
Shannon, R.V. (1985) Threshold and loudness functions for pulsatile stimulation of cochlear implants. Hear. Res. 18, 135-143.

Shannon, R.V. (1992) Temporal modulation transfer functions in patients with cochlear implants. J. Acoust. Soc. Am. 91, 21562164.

Shannon, R.V. (1993) Reply to: 'Comment on Temporal modulation transfer functions in patients with cochlear implants' [J. Acoust. Soc. Am 93, 1649-1650 (1993)]. J. Acoust. Soc. Am. 93, 16511652.

Skinner, M.W., Holden, L.K., Holden, T.A., Dowell, R.C., Seligman, P.M., Brimacombe, J.A. and Beiter, A.L. (1991) Performance of postlinguistically deaf adults with the wearable speech processor (WSP III) and mini speech processor (MSP) of the nucleus multielectrode cochlear implant. Ear. Hear. 12, 3-22.

Turner, C.W. and Nelson, D.A. (1982) Frequency discrimination in regions of normal and impaired sensitivity. J. Speech. Hear. Res. $25,34-41$.

Van Tasell, D.J., Soli, S.D., Kirby, V.M. and Widin, G.P. (1987) Speech waveform envelope cues for consonant recognition. J. Acoust. Soc. Am. 82, 1152-1161.

Van Tasell, D.J., Greenfield, D.G., Logemann, J.J. and Nelson, D.A. (1992) Temporal cues for consonant recognition: Training, talker generalization, and use in evaluation of cochlear implants. $\mathbf{J}$. Acoust. Soc. Am. 92, 1247-1257.

Viemeister, N.F. (1979) Temporal modulation transfer functions based upon modulation thresholds. J. Acoust. Soc. Am. 66, 1364-1380.

Zeng, F.G. and Shannon, R.V. (1992) Loudness balance between electrical and acoustic stimulation. Hear. Res. 60, 231-235. 